International Journal of Wireless \& Mobile Networks (IJWMN) Vol. 5, No. 1, February 2013

\title{
Performance Comparison of MIMO Systems over AWGN and Rician Channels with Zero Forcing Receivers
}

\author{
Navjot Kaur and Lavish Kansal \\ Lovely Professional University, Phagwara, \\ E-mails: er.navjot21@gmail.com, lavish.15911@lpu.co.in
}

\begin{abstract}
Multiple-Input Multiple-Output (MIMO) systems have been emerged as a technical breakthrough for high-data-rate wireless transmission. The performance of MIMO system can be improved by using different antenna selection so as to provide spatial diversity. In this paper, the performance of MIMO system over AWGN (Additive White Gaussian Noise) and Rician fading channels with ZF receiver is analyzed using different antenna configurations. The bit error rate performance characteristics of ZeroForcing (ZF) receiver is studied for M-PSK (M-ary Phase Shift Keying) modulation technique using $A W G N$ and Rician channels for the analysis purpose and their effect on BER (Bit Error Rate) have been presented.
\end{abstract}

Keywords - MIMO, spatial diversity, AWGN, Rician, fading, ZF, antenna, BER, M-PSK.

\section{INTRODUCTION}

MIMO systems make use of multiple antennas at the transmitter and receiver so as to increase the data rates by means of spatial diversity. So MIMO systems are well-known in wireless communications for high data rates. [1] The capacity of wireless systems can be increased by varying the number of antennas.

The two primary reasons for using wireless communication over wired communication:

- First is multi-path fading i.e. the variation of the signal strengths due to the various obstacles like buildings, path loss due to attenuation and shadowing [2].

- Second, for the wireless users, the transmission media is air as compared to the wired communication where each transmitter-receiver pair is considered as an isolated point-topoint link.

MIMO system utilizes the feature of spatial diversity by using spatial antennas in a dense multipath fading environment which are separated by some distance [3]. MIMO systems are implemented to obtain diversity gain or capacity gain to avoid signal fading. The idea to improve the link quality (BER) or data rate (bps) is the basic consideration behind the development of MIMO systems by using multiple TX/RX antennas [4]. The core scheme of MIMO is space-time coding (STC). The two main functions of STC: diversity \& multiplexing. The maximum performance needs tradeoffs between diversity and multiplexing. 
International Journal of Wireless \& Mobile Networks (IJWMN) Vol. 5, No. 1, February 2013

MIMO system employs various coding techniques for multiple antenna transmissions have become one of the desirable means in order to obtain high data rates over wireless channels [5]. However, of considerable concern is the increased complexity incurred in the implementation of such systems. MIMO antenna systems are used in recent wireless communications like WiMAX, IEEE 802.11n and 3GPP LTE etc.

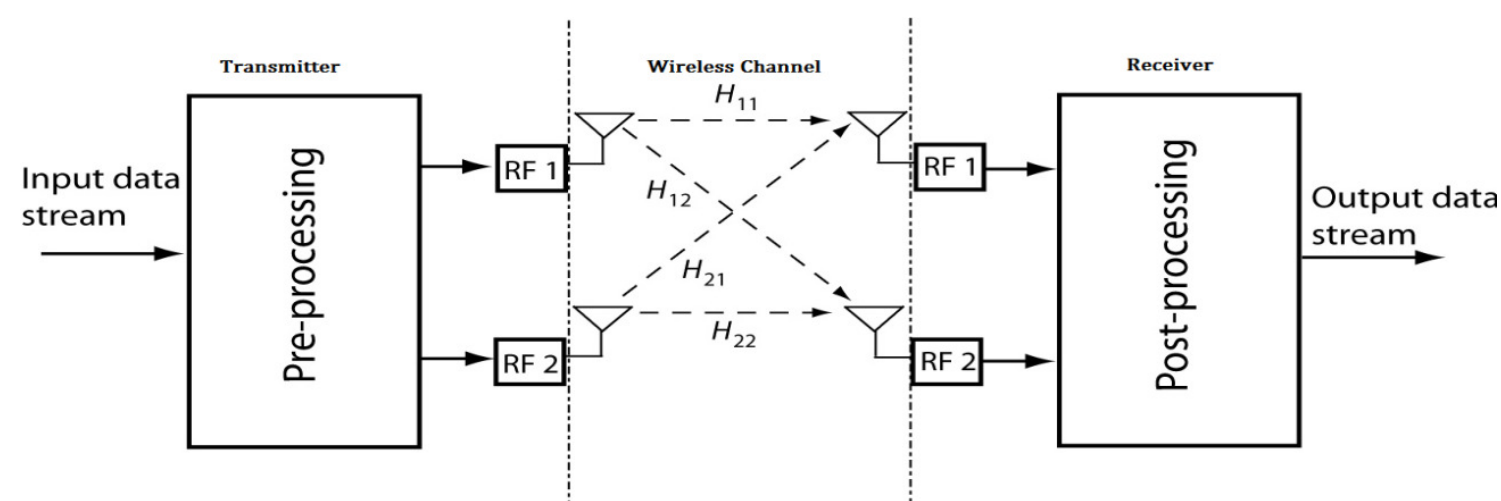

Fig. 1.1: MIMO System (2X2 MIMO Channel)

A. I. Sulyman [6] describes the performance of MIMO systems over nonlinear fading channels. The effects of antenna selection on its performance are also considered. The author has derived expressions for the PWEP performance of space-time trellis coding nonlinear Rayleigh fading channel. With the variation in the antenna selection at the receiver side, the performance degradation due to nonlinear fading channel reduces.

The comparison of MIMO with conventional Single-Input Single-Output (SISO) technology was discussed by S. G. Kim et. al [7]. The authors discussed that the MIMO system enhances the link throughput and also improves the spectral efficiency. The authors analyzed the BER performance of MIMO systems for M-PSK using ZF receiver over various fading channels in the presence of practical channel estimation errors.

C. Wang [8] explains the approach to increase the capacity of MIMO systems by employing spatial multiplexing. Maximum likelihood (ML) receiver achieves optimal performance whereas the linear receivers like Zero-Forcing (ZF) receiver provide sub-optimal performance. But Zero- Forcing receiver also offers significant reduction in computational complexity with performance degradation in tolerable limits.

A simple transmit diversity scheme comprises of two transmit antennas and one receive antenna was presented by X. Zhang et. al [9]. It provides the same spatial diversity order as that can be achieved by maximal-ratio receiver combining (MRRC) which makes use of one transmit antenna and two receive antennas.

A. Lozano et. al [10] compared the transmit diversity vs. spatial multiplexing in modern MIMO systems. Antenna diversity is a preferred weapon used by mobile wireless systems against the effect of fading. The prevalence of MIMO has opened the door for a much more effective use of antennas: spatial multiplexing.

The rich-scattering wireless channel is capable of enormous theoretical capacities if the multipath is properly exploited as per the researches done in the field of Information theory. P. 
International Journal of Wireless \& Mobile Networks (IJWMN) Vol. 5, No. 1, February 2013

W. Wolniansky et. al [11], described an architecture of wireless communication known as VBLAST (Vertical Bell Laboratories Layered Space-Time) that has been implemented in realtime environment.

An efficient implementation of space-time coding for the broadband wireless communications is presented by R. S. Blum et. al [12]. The authors presented the improved performance of MIMO-OFDM systems and diversity gains of a space time (ST) coding system through the type of trellis codes used in non-linear fading channel environment. The developed simulator for predicting the performance of a space time (ST) coded MIMO-OFDM system under different trellis coding and channel conditions is demonstrated.

The performance analysis of the low-cost effective MIMO system that employs the spatial multiplexing at the transmitter and zero-forcing processing at the receiver in multiuser scheduling systems was discussed by C. Chen [13]. By incorporating the mathematical tool of order statistics, the author derived the PDFs of effective sub channel output SNRs for a variety of scheduling algorithms. These expressions are used to derive the closed-form formulas. The closed-form expressions allow efficient numerical evaluations to characterize the capacity gain of this suboptimal transmission strategy under a number of practical scheduling policies requiring scalar or vector feedback. The results validate the elegant marriage of the zero-forcing receiver and scheduling technique as an economical approach to achieve higher data rates for next-generation wireless communications.

N. S. Kumar et. al [14], investigated about the three types of equalizer for MIMO wireless receivers. The authors discussed about a fixed antenna MIMO antenna configuration and compare the performance with all the three types of equalizer based receiver namely ZF, ML, and MMSE. BER performance of ML Equalizer is superior to zero forcing Equalizer and Minimum Mean Square Equalizers. It is inferred that the ML equalizer is the best of the three equalizers based on the mathematical modeling and the simulation results.

In this paper, the performance analysis of MIMO systems over AWGN and Rician channels using ZF receivers are presented. AWGN channel is a channel which has flat frequency response. It is known as universal channel model used for analyzing modulation schemes. In this, channel adds a white Gaussian noise to the signal passing through it. When there is line of sight, direct path is normally the strongest component goes into deeper fade compared to the multipath components. This kind of signal is approximated by Rician distribution.

\section{BENEFITS OF MIMO SYSTEMS}

\section{Spatial multiplexing}

Spatial multiplexing which comprises of number of transmit-receive antenna pairs tend to increase the transmission rate (or capacity) for the same bandwidth without any additional power expenditure. The increase in the transmission rate is proportional to the number of transmit-receive antenna pairs.

\section{Interference reduction and avoidance}

Multiple users which shares time and frequency resources result in interference in wireless networks. Interference may be mitigated in MIMO systems by exploiting the spatial dimension 
International Journal of Wireless \& Mobile Networks (IJWMN) Vol. 5, No. 1, February 2013

to increase the separation between users. To improve the coverage and range of a wireless network, there is need of interference reduction and avoidance.

\section{Array gain}

The coherent combining effect of multiple transmitting and receiving antennas tends to achieve good array gain at the receiver. This average increase in the SNR at the receiver requires perfect channel knowledge either at the transmitter or receiver or both.

\section{Diversity gain}

Multipath fading is the most significant problem in wireless communications due to various obstacles like building, scattering, reflection etc. In a fading channel, signal experiences fade (i.e the fluctuation in the signal strength). The channel is in deep fade when there is a significant drop in the signal power that gives rise to high BER. The diversity is used to so as to combat fading as much as it can.

Table 1.1: Benefits of MIMO system

\begin{tabular}{|c|c|c|}
\hline $\begin{array}{c}\text { MIMO } \\
\text { TECHNIQUE }\end{array}$ & BENEFITS & BEST CONDITIONS \\
\hline $\begin{array}{c}\text { SPATIAL } \\
\text { MULTIPLEXING }\end{array}$ & $\begin{array}{c}\text { Increases the throughput of } \\
\text { the system }\end{array}$ & $\begin{array}{c}\text { Best performance is achieved at low } \\
\text { velocity near to the base station (strong } \\
\text { signal) }\end{array}$ \\
\hline $\begin{array}{l}\text { TRANSMIT } \\
\text { DIVERSITY }\end{array}$ & $\begin{array}{l}\text { Increases the range by } \\
\text { countering fading (less } \\
\text { possibility of errors) } \\
\text { usually at base station }\end{array}$ & $\begin{array}{l}\text { Good when beam forming is not } \\
\text { appropriate }\end{array}$ \\
\hline $\begin{array}{l}\text { RECEIVE } \\
\text { DIVERSITY }\end{array}$ & $\begin{array}{l}\text { Increases the range by } \\
\text { countering fading (less } \\
\text { possibility of errors) } \\
\text { usually at mobile station }\end{array}$ & $\begin{array}{c}\text { Advantage over single antenna under all } \\
\text { conditions }\end{array}$ \\
\hline BEAMFORMING & $\begin{array}{c}\text { Increases the range at base } \\
\text { station }\end{array}$ & $\begin{array}{c}\text { Works best at relatively low velocity } \\
\text { when distance is extremely large (cell } \\
\text { edge). }\end{array}$ \\
\hline
\end{tabular}

\section{MODULATION TECHNIQUE}

Modulation is the process of superimposing a low frequency information signal over a high frequency carrier signal so that its transmission is possible over a long distance. Modulation can be analog and digital type. Digital modulation maps the digital information over analog carrier 
International Journal of Wireless \& Mobile Networks (IJWMN) Vol. 5, No. 1, February 2013

so as to transmit it over the channel. Every digital communication system has a modulator in the transmitter side and a demodulator in the receiver side. Every transmitter has a modulator that performs the task of modulation. Every $r$

eceiver has a demodulator to perform the inverse process of modulation, called demodulation, so as to recover the transmitted digital information.

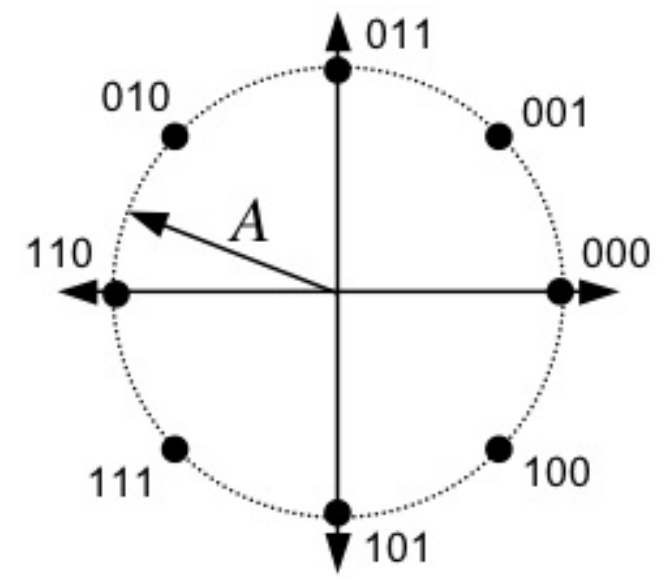

Fig. 1.2: Signal Space Diagram for 8-PSK

The M-ary PSK modulation yields circular constellation as the amplitude of the transmitted signals remains constant as shown in Fig. 1.2.

The signal set for M-ary Phase-shift keying (M-PSK) can be represented as:

$$
X_{i}(t)=\frac{\sqrt{2 E_{s}}}{T_{s}} \cos \left(2 \pi * f_{c \tau}+\frac{2(i-1)}{M}\right) \quad i=1,2, \ldots . M \& 0<<T_{s}
$$

where $E_{s}$ represents the signal energy per symbol, $T_{s}$ represents the symbol duration and $f_{c \tau}$ represents the carrier frequency.

This phase of the carrier changes for different possible values of $M$ as follows:

$$
\theta=2(i-1)^{\pi / M} \quad i=1,2, \ldots . M
$$

\section{CHANNELS USED}

Communication channels can be classified as fast and slow fading channels. In a fast channel, the impulse response changes approximately at the symbol rate of the communication system, whereas in a slow fading channel, it does not changes so frequently. Rather it stays unchanged for several symbols. In this paper, the performance analysis of MIMO system is discussed over the AWGN channel and Rician channel.

- AWGN channel: It is a channel used for analyzing modulation schemes by adding a white Gaussian noise to the signal passing through it. This channel's amplitude frequency response is flat and phase frequency response is linear for all frequencies. The modulated signals pass through it without any amplitude loss and phase distortion. So in such a case, fading does not exist but the only distortion that exists is introduced by the AWGN. The received signal is simplified to 
International Journal of Wireless \& Mobile Networks (IJWMN) Vol. 5, No. 1, February 2013

$$
r(t)=x(t)+n(t)
$$

where $n(t)$ represents the noise.

- Rician channel: When there is line of sight, direct path is normally the strongest component goes into deeper fade compared to the multipath components. This kind of signal is approximated by Rician distribution. As the dominating component run into more fade the signal characteristic goes from Rician to Rayleigh distribution. The signal characteristic goes from Rician to Rayleigh distribution as the dominating component run into more fade in multi-path fading.

$$
p(r)=\frac{r}{\sigma^{2}} e^{-\frac{\left(r^{2}+A^{2}\right)}{2 \sigma^{2}}} \text { I }\left(\frac{A r}{\sigma^{2}}\right) \quad \text { for }(A \geq 0, r \geq 0)
$$

Where A denotes the peak amplitude (value) of the dominant signal and $\mathrm{I}_{0}[$.$] is the$ modified Bessel function of zero-order.

\section{MIMO SYSTEM MODEL}

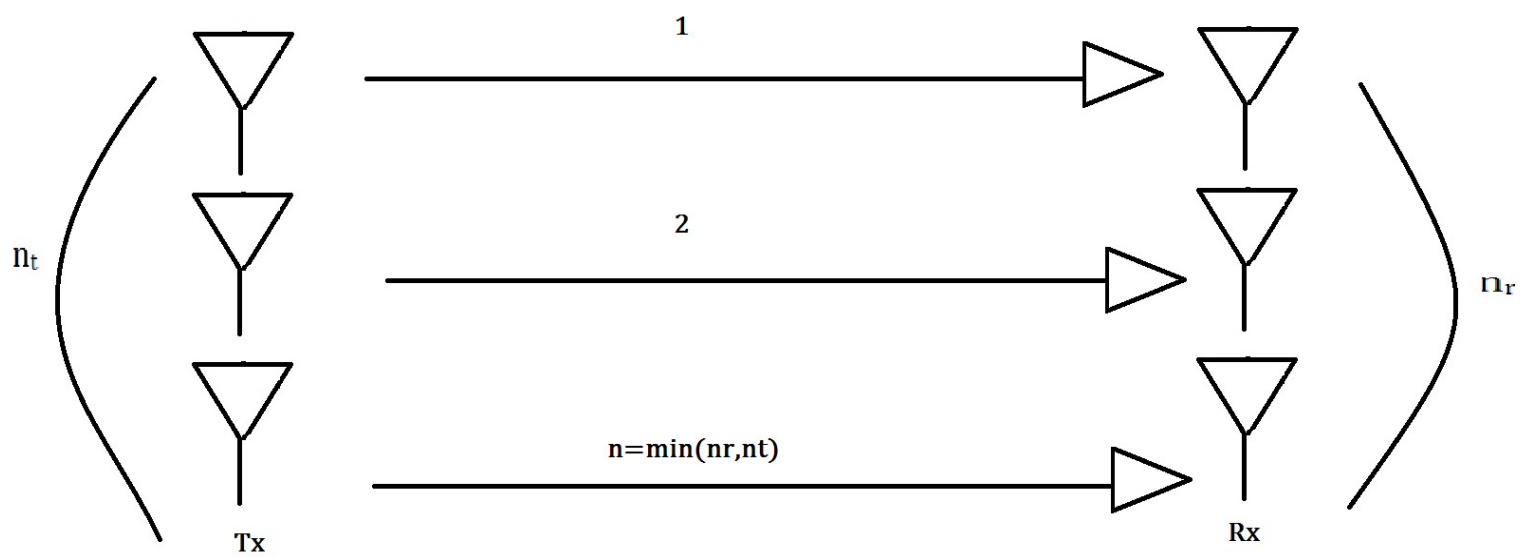

Fig. 1.3: MIMO channel as n SISO sub-channels

The MIMO channel is represented in Fig. 1.3 with an antenna array with $n_{t}$ elements at the transmitter and an antenna array with $n_{r}$ elements at the receiver is considered. The impulse response of the channel is $\mathrm{h}_{\mathrm{ij}}(\tau, \mathrm{t})$ between the $\mathrm{j}^{\text {th }}$ transmitter element and the $\mathrm{i}^{\text {th }}$ receiver element. The MIMO channel can then be described by the $n_{r} X n_{t} H(\tau, t)$ matrix:

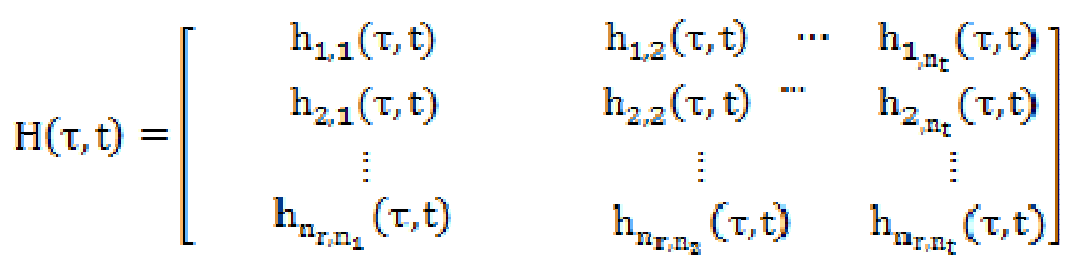


International Journal of Wireless \& Mobile Networks (IJWMN) Vol. 5, No. 1, February 2013

The matrix elements are complex numbers. These elements have dependency on the attenuation and phase shift that the wireless channel introduces delay $\tau$ to the received signal reaching at the receiver.

The input-output relation of the MIMO system can be expressed as follows:

$$
y(t)=H(\tau, t) \otimes s(t)+u(t)
$$

where ? denotes convolution, $s(t)$ is a $n_{t} X 1$ vector corresponding to the $n_{t}$ transmitted signals, $y(t)$ is a $n_{r} X 1$ vector corresponding to the $n_{r}$ and $u(t)$ is the additive white noise.

\section{ZERO FORCING EQUALIZER}

Zero Forcing Equalizer was first proposed by Robert Lucky, is a linear receiver used in communication systems. This equalizer inverts the frequency response of the channel to the received signal so as to restore the signal before the channel. This receiver is called Zero Forcing as it brings down the ISI to zero [5]. The frequency response of channel is assumed to be $\mathrm{F}(\mathrm{f})$ and $\mathrm{C}(\mathrm{f})$ for the zero forcing equalizer, then this equalizer is constructed such that $\mathrm{C}(\mathrm{f})=$ $1 / \mathrm{F}(\mathrm{f})$. Thus this combination of channel and equalizer gives a flat frequency response and linear phase.

The received signal can be represented by using the linear model as:

$$
\mathrm{y}=\mathrm{Hx}+\mathrm{n}
$$

A 2x2 MIMO channel can be represented in matrix notation as follows:

$$
\left(\begin{array}{l}
y_{1} \\
y_{2}
\end{array}\right)=\left(\begin{array}{ll}
h_{1,1} & h_{1,2} \\
h_{2,1} & h_{2,2}
\end{array}\right)\left(\begin{array}{l}
x_{1} \\
x_{2}
\end{array}\right)+\left(\begin{array}{l}
n_{1} \\
n_{2}
\end{array}\right)
$$

The signal received on the first receive antenna can be expressed as:

$$
\mathrm{y}_{1}=\mathrm{h}_{1,1} \mathrm{x}_{1}+\mathrm{h}_{1,2} \mathrm{x}_{2}+\mathrm{n}_{1}=\left[\begin{array}{ll}
\mathrm{h}_{1,1} & \mathrm{~h}_{1,2}
\end{array}\right]\left[\begin{array}{l}
\mathrm{x}_{1} \\
\mathrm{x}_{2}
\end{array}\right]+\mathrm{n}_{1}
$$

The signal received on the second receive antenna can be expressed as:

$$
y_{2}=h_{2,1} x_{1}+h_{2,2} x_{2}+n_{2}=\left[\begin{array}{ll}
h_{2,1} & h_{2,2}
\end{array}\right]\left[\begin{array}{l}
x_{1} \\
x_{2}
\end{array}\right]+n_{2}
$$

where

$\mathrm{x}_{1}$ and $\mathrm{y}_{1}$ is the transmitted and received symbol on the first antenna, $\mathrm{x}_{2}$ and $\mathrm{y}_{2}$ is the transmitted and received symbol on the second antenna, $\mathrm{h}_{1,1}$ is the channel from $1^{\text {st }}$ transmit antenna to the $1^{\text {st }}$ receive antenna, $\mathrm{h}_{1,2}$ is the channel from $2^{\text {nd }}$ transmit antenna to the $1^{\text {st }}$ receive antenna, $\mathrm{h}_{2,1}$ is the channel from $1^{\text {st }}$ transmit antenna to the $2^{\text {nd }}$ receive antenna, $\mathrm{h}_{2,2}$ is the channel from $2^{\text {nd }}$ transmit antenna to the $2^{\text {nd }}$ receive antenna, and $\mathrm{n}_{1}, \mathrm{n}_{2}$ are the noise on $1^{\text {st }}$ and $2^{\text {nd }}$ receive antennas. 
International Journal of Wireless \& Mobile Networks (IJWMN) Vol. 5, No. 1, February 2013

\section{SIMULATED RESULTS}

In this section, the BER analysis of MIMO system structure is done for M-PSK Modulation techniques over AWGN and Rician fading channels using Space-Time Block Coding (STBC) structure. The BER analysis of MIMO system is done for M-PSK modulation for different values of M. Here the value of M selected can be 32, 64, 128, 256, 512 and 1024 over both the fading channels.

\section{(A)M-PSK over AWGN channel}

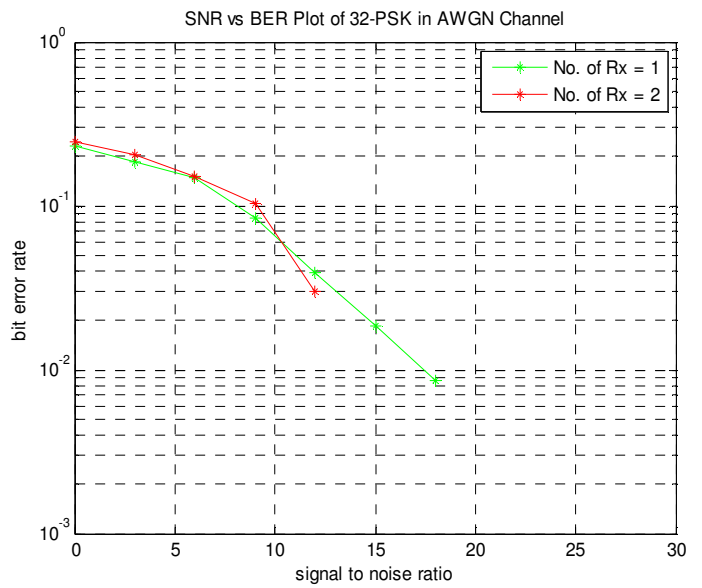

(a) 32-PSK

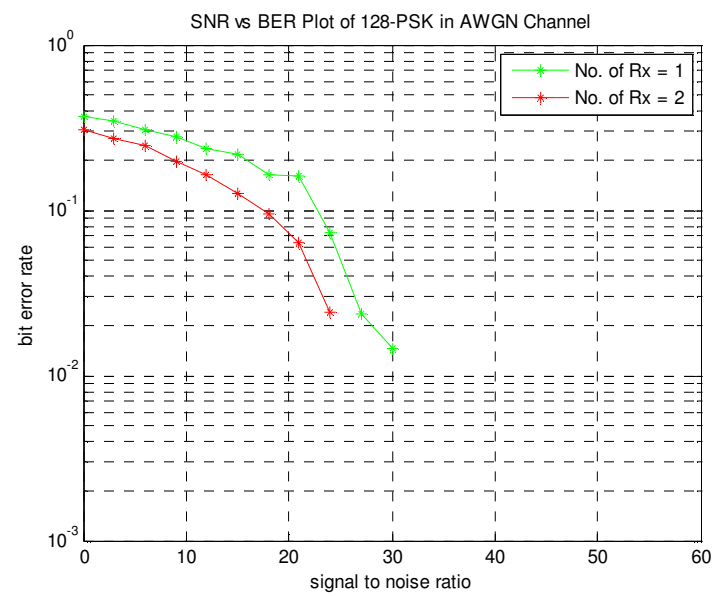

(c) 128-PSK

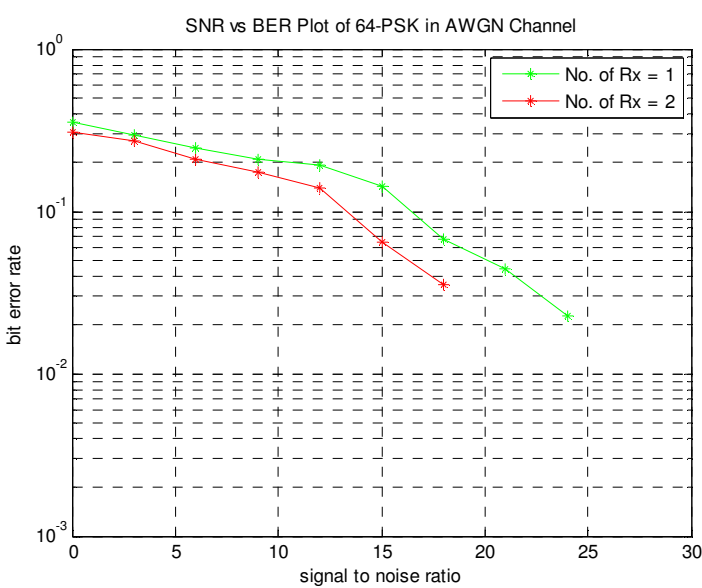

(b) 64-PSK

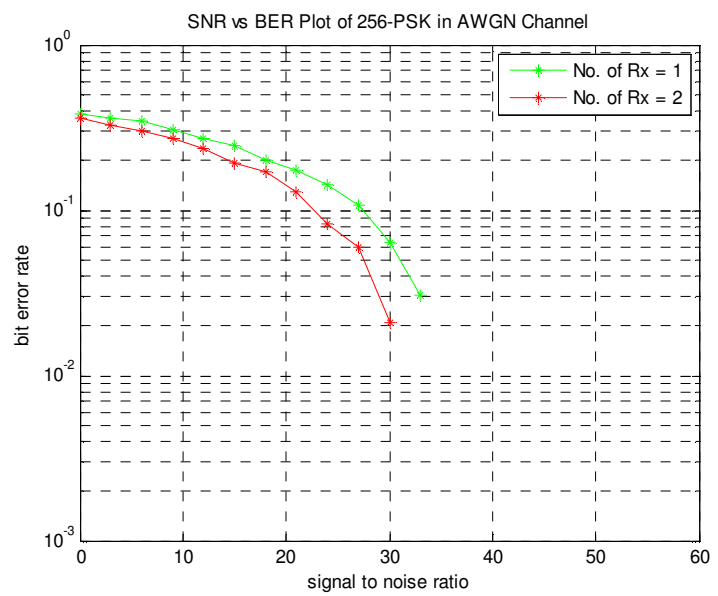

(d) 256-PSK 
International Journal of Wireless \& Mobile Networks (IJWMN) Vol. 5, No. 1, February 2013

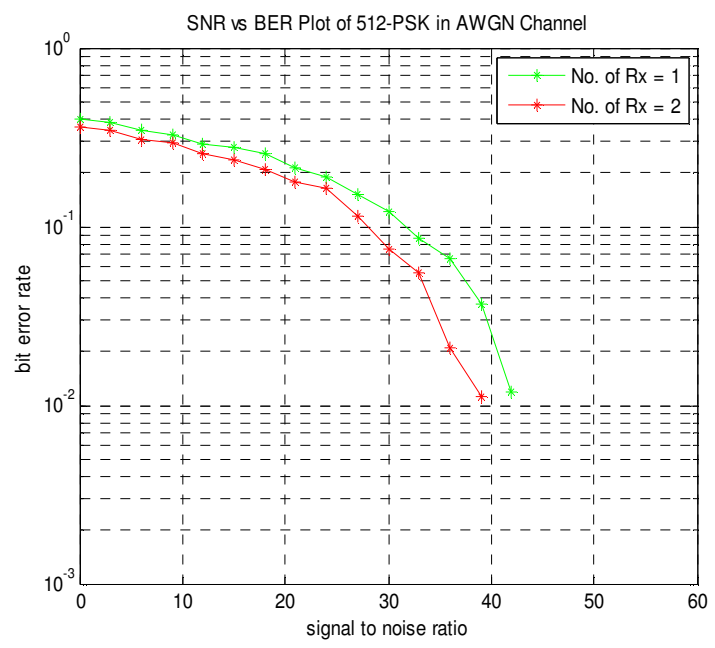

(e) 256-PSK

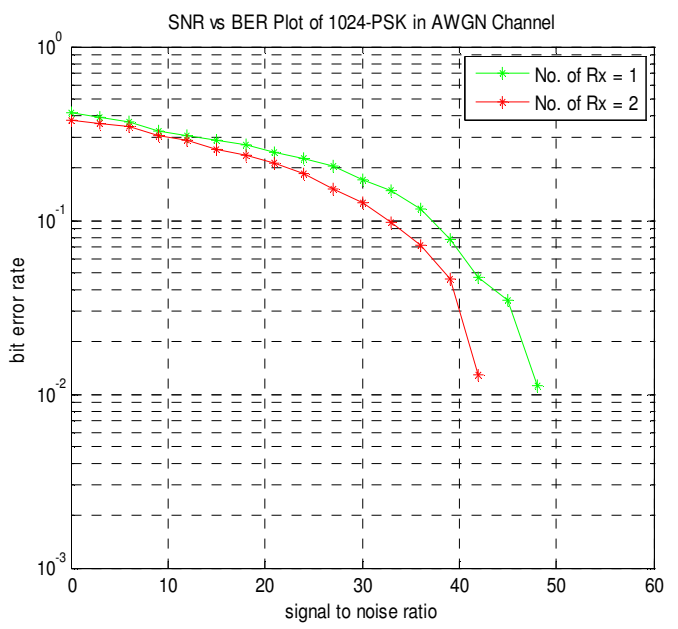

(f) 1024-PSK

Fig. 1.4: SNR vs BER plots using M-PSK over AWGN channel for different values of $M$

In Fig. 1.4 (a) - (f), the SNR vs BER plots using M-PSK over AWGN channel for MIMO system are presented for different values of ' $M$ ' employing different antenna configurations. It can be concluded from the graphs that with the increase in number of receiving antennas, the BER keeps on decreasing due to space diversity in MIMO and thus the system proposed over here provide better BER performance in comparison to the other antenna configurations.

\section{(B) M-PSK over Rician channel}

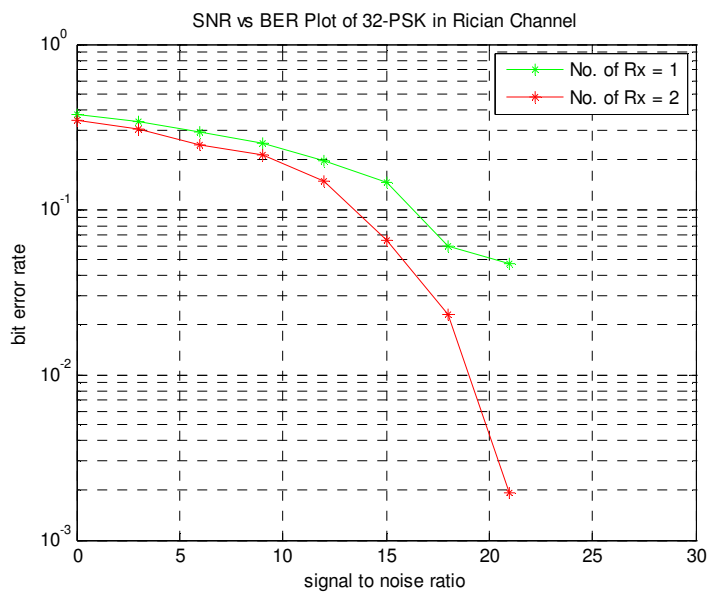

(a) 32-PSK

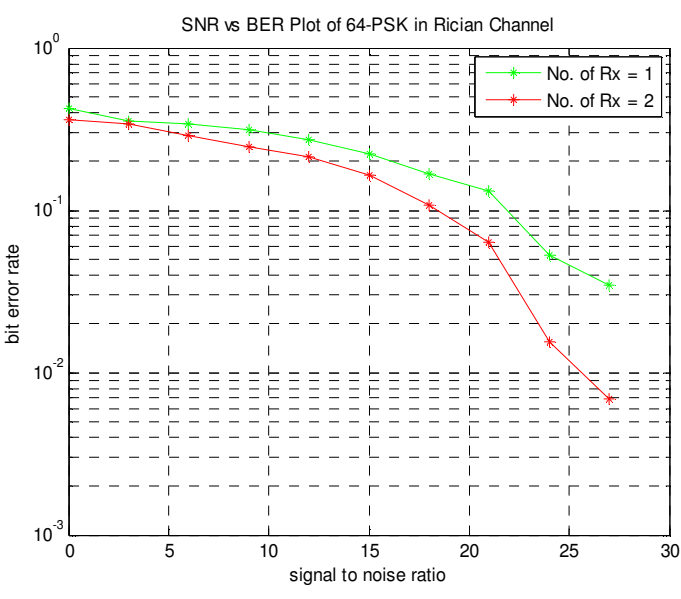

(b) 64-PSK 
International Journal of Wireless \& Mobile Networks (IJWMN) Vol. 5, No. 1, February 2013

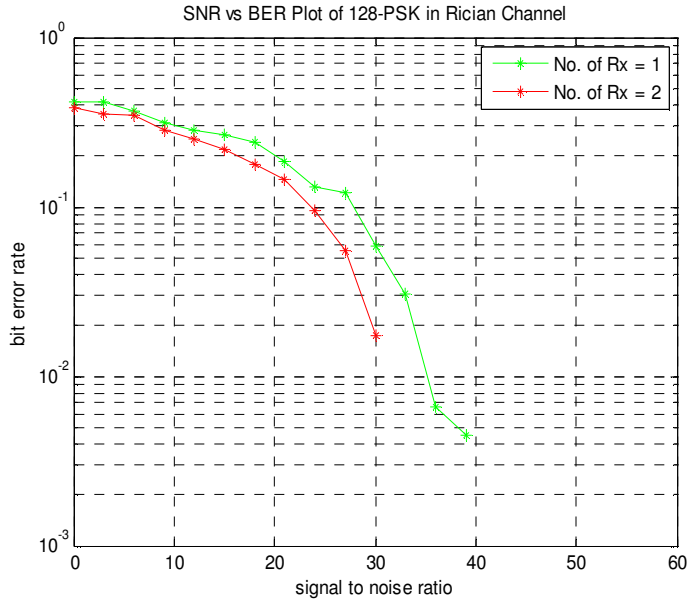

(c) 128-PSK

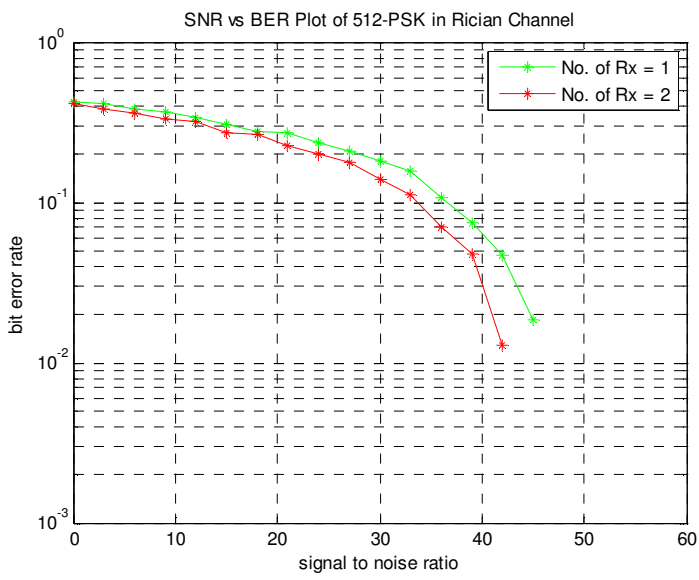

(e) 512-PSK

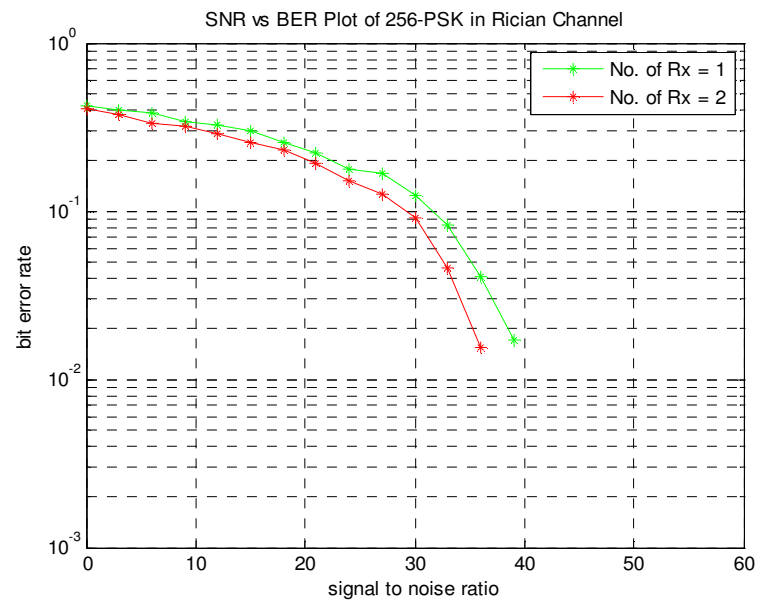

(d) 256-PSK

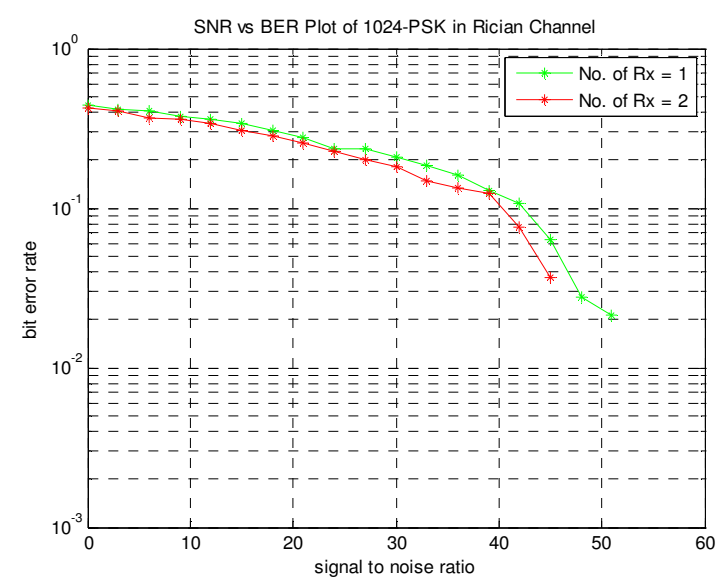

(f) 1024-PSK

Fig. 1.5: SNR vs BER plots using M-PSK over Rician channel for different values of $M$

The SNR vs BER plots using M-PSK over Rician channel for MIMO system are presented for different values of ' $M$ ' employing different antenna configurations are presented in Fig. 1.5 (a) - (f). From the graphs, it can be seen that if there is increase in the number of receiving antennas in MIMO system then the BER keeps on decreasing due to space diversity. Thus this system provides better BER performance as compared to the other antenna configurations.

\section{CONCLUSION}

In this paper, SNR vs. BER plots for M-PSK over AWGN and Rician fading channels for MIMO system employing different antenna configurations are presented. It can be concluded that in MIMO system, the BER keeps on decreasing due to space diversity as we goes on increasing the number of receiving antennas and the proposed system provide better BER 
International Journal of Wireless \& Mobile Networks (IJWMN) Vol. 5, No. 1, February 2013

performance. But BER is greater in Rician channel as compared to that of AWGN channel. Also as we goes on increasing the value of M for M-PSK i.e the no. of constellation points in the constellation diagram, the BER is also increasing. This increase in BER is due to the fact that as increase the size of constellation diagram the spacing in between different constellation point will keep on decreasing, which results in decreasing the width of decision region for each constellation point which in turn makes the detection of the signal corresponding to the constellation point much tougher. Due to this fact the BER is increasing as we goes on increasing the number of points in constellation diagram.

\section{REFERENCES}

[1] P. Sanghoi \& L. Kansal, “Analysis of WIMAX Physical layer Using Spatial Diversity”, International Journal of Computer Application, Vol. 44, Issue 5, 2012.

[2] L. Kansal, A. Kansal \& K. Singh, "BER Analysis of MIMO-OFDM Sytem Using OSTBC Code Structure for M-PSK under Different fading Channels", International Journal of Scientific \& Engineering Research, Vol. 2, Issue 11, 2011.

[3] P. Sanghoi \& L. Kansal, "Analysis of WIMAX Physical layer Using Spatial Diversity under different Fading Channels", International Journal of Computer Application, Vol. 44, Issue 20, 2012.

[4] S. Alamouti, "A simple transmit diversity technique for wireless communications", IEEE Journal on Selected Areas of Communication, Vol. 16, Issue 8, pp. 1451-1458, 1998.

[5] V. Tarokh, H. Jafarkhani \& A. R. Calderbank, "Space-time block codes from orthogonal designs", IEEE Transactions on Information Theory, Vol. 45, Issue 5, pp. 1456-1467, 1999.

[6] A. I. Sulyman, "Performance of MIMO Systems With Antenna Selection Over Nonlinear Fading Channels", IEEE Journal of Selected Topics in Signal Processing, Vol. 2, Issue 2, pp. 159-170, 2008.

[7] S. G. Kim, D. Yoon, Z. Xu \& S. K. Park, "Performance Analysis of the MIMO Zero-Forcing Receiver over Continuous Flat Fading Channels", IEEE Journal of Selected Areas in Communications, Vol. 20, Issue 7, pp. 324 - 327, 2009.

[8] C. Wang, "On the Performance of the MIMO Zero-Forcing Receiver in the Presence of Channel Estimation Error", IEEE Transactions on Wireless Communications, Vol. 6, Issue 3, pp. $805-810$, 2007.

[9] X. Zhang, Z. Lv \& W. Wang, "Performance Analysis of Multiuser Diversity in MIMO Systems with Antenna Selection", IEEE Transactions on Wireless Communications, Vol. 7, Issue 1, pp. 15-21, 2008.

[10] A. Lozano \& N. Jindal, "Transmit Diversity vs. Spatial Multiplexing in Modern MIMO Systems", IEEE Transactions on Wireless Communications, Vol. 9, Issue 1, pp. 186-197, 2010.

[11] P. W. Wolniansky, G. J. Foschini, G. D. Golden \& R. A. Valenzuela, "V-Blast: An architecture for realizing very high data rates over the rich-scattering channel", International Symposium on Signals, Systems and Electronics, pp. 295-300, 1998.

[12] R. S. Blum, Y. Li, J. H. Winters \& Q. Yan, "Improved Space-Time Coding for MIMO-OFDM Wireless Communications", IEEE Transaction on Communications, Vol. 49, Issue 11, pp. 1873$1878,2001$.

[13] C. Chen, "Performance Analysis of Scheduling in Multiuser MIMO Systems with Zero-Forcing Receivers", IEEE Journal of Selected Areas in Communications, Vol. 25, Issue 7, pp. 1435-1445, 2007. 
International Journal of Wireless \& Mobile Networks (IJWMN) Vol. 5, No. 1, February 2013

[14] N. S. Kumar, G. J. Foschini, G. D. Golden \& R. A. Valenzuela, "Bit Error Rate Performance Analysis of ZF, ML and MMSE Equalizers for MIMO Wireless Communication Receiver", European Journal of Scientific Research, Vol. 59, Issue 4, pp. 522-532, 2011.

\section{Authors}

Navjot Kaur was born in Jalandhar. She received her B.Tech degree in Electronics and Communication Engineering from Lovely Institute of Technology, Phagwara, Punjab Technical University, Jalandhar, in 2008, and presently pursuing M.Tech degree in Electronics and communication engineering from Lovely Professional University, Phagwara, India. Her research interests include MIMO systems and wireless systems.

Lavish Kansal was born in Bathinda. He received his B.Tech degree in Electronics and Communication Engineering from PTU, Jalandhar in 2009 and M.E. degree in Electronics and Communication Engineering from Thapar University, Patiala in 2011.He is working as Assistant Professor in the department of Electronics and communication Engineering, Lovely Professional University, Phagwara, India. He has published 15 papers in International journals. His research area includes Digital Signal Processing, Digital Communication \& Wireless Communication.
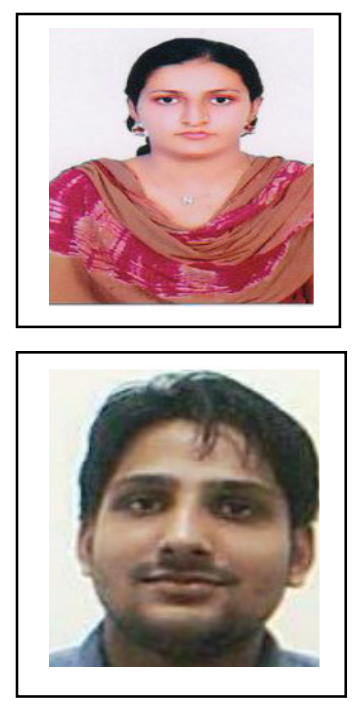